\title{
Ion-scale structure in Mercury's magnetopause reconnection diffusion region
}

\author{
Daniel J. Gershman ${ }^{1,2}$, John C. Dorelli ${ }^{2}$, Gina A. DiBraccio ${ }^{2}$, Jim M. Raines ${ }^{3}$, James A.
} Slavin $^{3}$, Gangkai Poh $^{3}$, and Thomas H. Zurbuchen ${ }^{3}$

(1) pepartment of Astronomy, University of Maryland, College Park, MD, 20742

(2)

(3) Climate and Space Sciences and Engineering, University of Michigan, Ann Arbor, Mighigan, USA, 48109

Th stre gth and time dependence of the electric field in a magnetopause diffusion region relate rate of magnetic reconnection between the solar wind and a planetary magnetrc field. Here, we use $\sim 150 \mathrm{~ms}$ measurements of energetic electrons from MESSENGER observed over Mercury's dayside polar cap boundary (PCB) to infer such small_cale changes in magnetic topology and reconnection rates. We provide the first dir asurement of open magnetic topology in flux transfer events at Mercury, stre Cirys thought to account for a significant portion of the open magnetic flux transport throughout the magnetosphere. In addition, variations in PCB latitude likely correspond to inte ittent bursts of $\sim 0.3-3 \mathrm{mV} / \mathrm{m}$ reconnection electric fields separated by $\sim 5-10 \mathrm{~s}$, resumg in average and peak normalized dayside reconnection rates of $\sim 0.02$ and $\sim 0.2$, respectively. These data demonstrate that structure in the magnetopause diffusion region at Nicreury occurs at the smallest ion scales relevant to reconnection physics.

Key Points:

(1) mangetic electrons at Mercury map magnetic topology at $\sim 150 \mathrm{~ms}$ (2) Jirstdirect observation of flux transfer event open-field topology at Mercury

(3) Mogylations of the reconnection rate at Mercury occur at ion kinetic scales

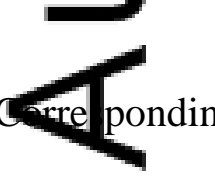

This is the author manuscript accepted for publication and has undergone full peer review but has not been through the copyediting, typesetting, pagination and proofreading process, which may lead to differences between this version and the Version of Record. Please cite this article as doi: 10.1002/2016GL069163

This article is protected by copyright. All rights reserved. 


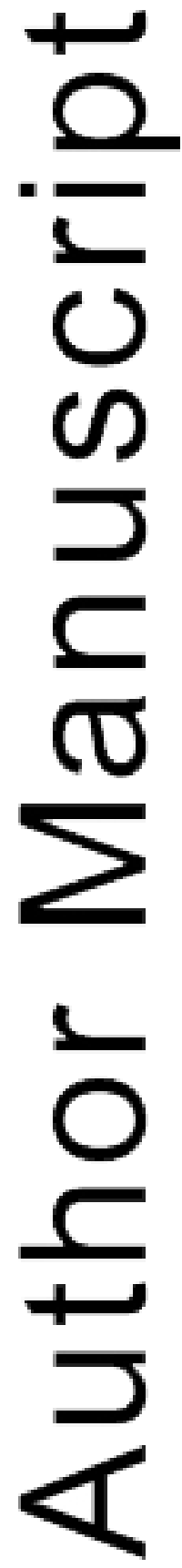

This article is protected by copyright. All rights reserved. 


\section{Introduction}

Magnetic reconnection is a ubiquitous process in space plasmas whereby magnetic topology is reconfigured and magnetic energy is converted into particle energy. The important physical processes relevant to this conversion take place within a diffusion regron, where ion and electron motions decouple from both each other and from the bad kgrond magnetic field, and where the 'frozen-in-field' approximation in space plasmasis no longer valid [Parker, 1957; Sweet, 1958]. In a planetary magnetosphere, manetic reconnection can occur at the dayside magnetopause, where the interplanetary maneth field (IMF) carried by the solar wind interacts with that of a planetary body. Here, the closed magnetic flux (i.e., connected to the planetary body only) is converted int 6 flux (i.e., connected to the planetary body and the solar wind). he topological boundary between the last closed field line and first open field line in the magnetosphere maps the magnetopause diffusion region to the polar cap boundary (PCB), which encloses all the planetary open magnetic flux. Magnetic reconamation at the magnetopause adds flux to the polar cap such that the dayside PCB mowes lower latitudes [Siscoe and Huang, 1985; Milan and Slavin, 2011]. Newly resanected flux is then convected over the polar cap by the magnetosheath flow and into magnetotail. Magnetic reconnection in the neutral sheet converts open magnetic flux from each pole into closed magnetic flux that eventually convects back towards the day vide magnetosphere. Observations of the time evolution of the PCB latitude can pro can insight into the time history of both the dayside and nightside reconnection rates. This voundary can be identified in situ via the presence of solar wind-borne particle preipitation along the open field [Winningham and Heikkila, 1974; Troshichev et al., 1996] omremotely from auroral emission observations [Feldstein and Starkov, 1967; Eat Akasofu, 1969], though precipitation from trapped energetic particles in closentreld regions can obscure these observations.

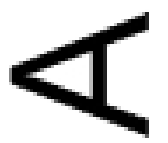


At Earth, a large ( 1000x) increase in the magnetic field (B) magnitude from the magnetopause to the polar cap limits changes in polar cap latitude due to newly reconnected magnetic flux $\left(<<1^{\circ} / \mathrm{MWb}\right)$. At Mercury, however, due to its comparatively weak internal magnetic field, a modest ( 2-3x) increase in the magnetic field strength fronthe magnetopause to the polar cap enables more measurable variations in the polar caplatit de $\left(\sim 10^{\circ} / \mathrm{MWb}\right)$. Furthermore, due to the absence of a stable radiation belt, Mercurv's PCB can be identified clearly and unambiguously through in situ solar ene particle (SEP) observations [Gershman et al., 2015]. Mercury's polar cap the efor provides a highly sensitive laboratory for the study of small-scale variations in magnetic topology and reconnection rate.

Directly observed discrete units of reconnected flux on the dayside are associated witnrmu transfer events (FTEs), which are helical structures that carry magnetic flux [Russell and Elphic, 1979]. Individual FTEs carry $\sim 1 \mathrm{MWb}$ and up to $\sim 0.1 \mathrm{MWb}$ of ma netic flux at Earth and Mercury, respectively [Slavin et al. 2010a,2012; Imber et al., 2014 Pecause the average open magnetic flux content at Mercury is only $\sim 2.5 \mathrm{MWb}$ [Joms et al., 2012], it has been suggested that showers of FTEs transport a significant portion of the open flux throughout the magnetosphere [Slavin et al. 2012; Imber et al. 24 However, no direct measurement of FTE topology that confirms their transport of open magnetic flux has yet been reported.

S. Here, we examine high cadence $(\sim 150 \mathrm{~ms})$ electron observations from the Fast Ima smy Plasma Spectrometer (FIPS) [Andrews et al., 2007] and magnetic field from the Magnetometer experiment [Anderson et al., 2007] on the MESSENGER spacecraft from traisits of the polar cap during strong SEP events. We provide the first direct mensurement of the magnetic topology in an FTE at Mercury demonstrating that they can ind open, with one end connected to the planet and the other to the interplanetary magretrc field. In addition, we will use PCB fluctuations with respect to invariant latitude $r$ the time history of the magnetopause reconnection electric field. 


\section{Data Selection and Processing}

Nominally, FIPS samples ions with energy-per-charge $(E / q)$ ratios between 10eV/e and $13.3 \mathrm{keV} / \mathrm{e}$ in $~ 10 \mathrm{~s}$ using 60 logarithmically spaced steps [Andrews et al., 20077. However, the thin $(\sim 1 \mathrm{~mm})$ aluminum shielding and use of microchannel plate detector for FIPS result in high sensitivity to the $>1 \mathrm{MeV}$ electrons and $>10 \mathrm{MeV}$ protons that bathe Mercury's magnetosphere during SEP events [Gershman et al., 2015]. Encretic protons have gyroradii that are large (e.g., 1000 km) compared to Mercury's ma hetopheric system (1 Mercury radius is $\sim 2400 \mathrm{~km}$ ). Consequently, they are not greatly deflected by the planetary magnetic field and their flux does not significantly ded across the magnetopause. However, energetic electrons, in particularly those precrpityting over the polar cap, have much smaller gyroradii $(\sim 1-10 \mathrm{~km})$ and consequently we observe sharp changes in the FIPS energetic particle-induced count rates of s detectors that correspond with magnetospheric boundaries.

When the energetic electron rates from this event are high compared to nominal
magneric thermal plasma countrates, we can substantially increase measurement timnesolution by using rates derived from the individual $E / q$ channels instead of an rage rate for each FIPS scan. Each 10s burst scan includes several seconds of high voltage ramp up such that there are small gaps between adjacent scans. Furthermore, althong the timing within a scan is known to millisecond precision, due to truncation of the cam time in the spacecraft telemetry, there is an absolute uncertainty of the start of eachsean by a fraction of a second. Finally, unphysical artifacts that arise from the FIPS pro essing of very high background signals manifest themselves as large, single $E / q$ channel-enhancements in the reported count rates. These electronic artifacts have been eliminat from the dataset. Despite these caveats, we can effectively use FIPS' indiviutul $E / q$ channel rates to provide a $\sim 150 \mathrm{~ms}$ proxy for changes in magnetic toperc gy. 
A close up of a magnetospheric FTE during an SEP event on 21 September 2012 demonstrates the ability of high-resolution FIPS measurements to reveal fine-scale magnetic topology in Figure 1. Magnetic field vectors for the duration of this FTE are shown in minimum variance coordinates [Sonnerup and Cahill, 1967; Slavin et al., 2010a], where the intermediate variance direction shows a relative core-field enlance hent of 20 nT and the maximum variance direction shows a corresponding bipolar signature indicative of helical structure embedded in the background manetospheric field. A high average minimum variance component ( $80 \mathrm{nT}$ ) indicates tha MESSENGER merely clipped the edge of this structure. Nonetheless, increased SEP electron fluxes relative to the closed-field magnetosphere persist for approximately $\sim 2 \mathrm{~s}$ that ospond with the observed center of the flux rope at 15:14:36 UT, indicating that its core ransports open magnetic flux. Smaller-time-scale ( 0.5 s) fluctuations in electron fluxes may further indicate a complex magnetic topology, although such signatures are convolved with finite-gyroradii effects of the precipitating electrons.

IIPS energetic electron fluxes relative to magnetosheath levels are shown for an SENent on 8 June 2011 in Figure 2. High energetic particle-induced countrates from bothectrons and ions are observed as MESSENGER flies over the open-field polar cap The rates fluctuate near the PCB denoted as the polar cap boundary layer (PCBL) until reaching a steady value of $\sim 0.5$ throughout the dayside closed-field region, to which ene electic elens have limited access. After crossing the magnetopause (MP) at 00:39 UT 1 Itto he dayside magnetosheath, the relative electron flux returns to $\sim 1$. In the PCBL, MESseNGER was approximately $\sim 1$ Mercury radius $\left(R_{\mathrm{M}}\right)$ away from the MercurySolr-Magnetospheric (MSM) origin [Anderson et al., 2011], i.e., on the sphere defining invarian latitude that is offset from the center of the planet by $\sim 0.2 R_{\mathrm{M}}$. The PCBL is obs over a $\sim 10^{\circ}$ range of invariant latitudes corresponding to $\sim 500 \mathrm{~km}$ in arc length on the sphere of $1 R_{\mathrm{M}}$. At the spacecraft location we further define a local spherical al nate system where $\hat{\boldsymbol{r}}$ points radially outward from the $M S M$-origin, $\widehat{\boldsymbol{\theta}}$ points in the 
direction of decreasing invariant latitude, and $\widehat{\boldsymbol{\phi}}$ completes the right-handed coordinate system and is oriented approximately duskward.

\section{Analysis and Discussion}

In a Dungey-like magnetospheric system, a newly reconnected parcel of plasma nea the magnetopause becomes magnetically connected to a parcel of plasma at the PCB and flaws over the polar cap with the magnetosheath flow. These parcels must remain matrically connected until additional magnetic reconnection (e.g., in the magnetotail) rec nfig Ires their magnetic topology. The PCBL is observed as MESSENGER alternatingly samples parcels of plasma that are magnetically connected and uncond to the interplanetary magnetic field. Through field-aligned 'Region 1' currents [Añers $n$ n et al., 2014], the reconnection electric field in the diffusion region $\left(\mathbf{E}_{\mathbf{R}} \approx E_{\mathrm{R}}\right.$ $\widehat{\boldsymbol{\phi}})$ maps to a dawn-dusk polar cap electric field $\left(\mathbf{E}_{\mathbf{p c}} \approx E_{p c} \widehat{\boldsymbol{\phi}}\right)$ that drives anti-sunward pla maconvection with velocity $\mathbf{v}_{\mathrm{pc}}=\left(\mathbf{E}_{\mathbf{p c}} \mathbf{x} \mathbf{B}_{\mathbf{p c}}\right) / B_{\mathrm{pc}}{ }^{2}$ [Slavin et al., 2009]. Here, in the nor ${ }^{\mathbf{2}}$ hemisphere, the direction of Mercury's dipole, $\mathbf{B}_{\mathrm{pc}}$ points planetward, i.e., in predommantly the $-\hat{\boldsymbol{r}}$ direction. Superimposed on this motion can be wave activity that creatoclocalized variations in $\mathbf{v}_{\mathbf{p c}}, \mathbf{E}_{\mathbf{p c}}$, and $\mathbf{B}_{\mathbf{p c}}$. We therefore consider the contributions affects to our observations: (1) back and forth wave motion of the PCB and (2) time-varying dayside magnetic reconnection. Magnetic reconnection in the magnetotail and absequent return of closed magnetic flux to the dayside will cause the PCB to con ract and move poleward, resulting in an underestimation of reconnected flux. However, tail loading (e.g., Slavin et al. [2010b]) and return convection processes can act as low pass filters for the circulation of magnetic flux in a magnetospheric system. Thests, which are observed to have the time scale of $10^{1}-10^{3} \mathrm{sec}$, are not likely to be oumpant on these very short (i.e., $10^{0} \mathrm{sec}$ ) timescales.

\footnotetext{
Steady anti-sunward motion of the magnetically connected magnetosheath and puar ap plasmas results in smooth poleward motion of the PCB with velocity $\mathbf{v}_{\mathrm{pc}}=$
} 
$\left(\mathbf{E}_{\mathbf{p c}} \mathrm{x} \mathbf{B}_{\mathbf{p c}}\right) / B_{\mathrm{pc}}{ }^{2}$ [Dungey, 1961]. Any disruption of this alignment due to PCB waves must produce a kink in the magnetic field, i.e., a change in $B_{\theta} / B$. Because of the close $(\sim 1000$ $\mathrm{km}$ ) proximity of the magnetopause to the spacecraft and planetary core [Anderson et al., 2014], kink angles of $\pm 15^{\circ}$ are required to reproduce apparent motions of the PCB prasmas per the $\sim 500 \mathrm{~km}$ arc of invariant latitude transited by the spacecraft during these varations.

- Alternatively, consider the illustration in Figure 3, which shows a top view of the ma netosphere. As MESSENGER descends out of the polar cap (Figure 3a), there is a sha $p$ reguction in measured energetic electron flux. If dayside reconnection occurs (Figure $3 \mathrm{~b}$ ) over some azimuthal distance across the magnetopause, $L_{\mathrm{MP}}$, a mapped dis 0 in the polar cap, $L_{\mathrm{PC}}$, will expand equatorward by a distance $D_{\mathrm{PC}}$ to accommpdate new open magnetic flux, i.e., $|\Phi| \approx\left|L_{P C} D_{P C} B_{P C}\right|$. If sufficient flux has been added to the polar cap, $D_{\mathrm{PC}}$ can grow large enough to overtake the spacecraft and opgr-field electron fluxes are measured. At a time $\triangle T$ later, MESSENGER then once againats the polar cap region. The duration of observed increased electron flux is definelas $\Delta t$. We can therefore estimate the distance $D_{\mathrm{PC}}$ as,

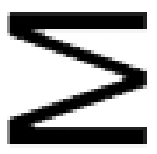

$$
D_{P C} \approx R_{M} \Delta \theta_{S C}-\left(\boldsymbol{v}_{P C} \cdot \widehat{\boldsymbol{\theta}}\right) \Delta \mathrm{t}
$$

wh $\theta_{S C}$ is the invariant latitude traversed by the spacecraft in time $\Delta T$. Because the pol $\mathrm{rca}$ plasma velocity is in the $-\widehat{\boldsymbol{\theta}}$ direction, the second term in equation (1) is positive. Therefore, anti-sunward convection increases our estimate of $D_{\mathrm{PC}}$. This cycle of entring and exiting the polar cap can repeat multiple times provided that dayside rotion continues with sufficient strength for the PCB to overtake the spacecraft (Figure $c$ ). Eventually, due to MESSENGER's orbital trajectory, the spacecraft will remain in the closed-field dayside magnetosphere until it crosses the dayside agn topause (Figure 3d). 
From Faraday's law of induction, the change of magnetic flux in the polar cap $(\Phi)$ due to dayside magnetic reconnection is equal to the integral of the electric field around the PCB, i.e., $\oint_{P C} \boldsymbol{E} \cdot d \boldsymbol{l}=-\frac{d \Phi}{d t}$. The right hand side of this equation can be estimated as,

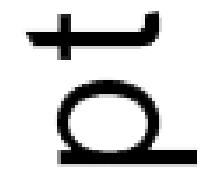

$$
-\frac{d \Phi}{d t} \approx-\frac{\left(\boldsymbol{B}_{P C} \cdot \hat{\boldsymbol{r}}\right) L_{P C} D_{P C}}{\Delta T}
$$

He,$\Delta T$ is used as the time over which flux was added to the polar cap. $\Delta T$ is an upper bound fro the time, which cannot be evaluated due to observations from a single spacenaft. Because Mercury's magnetic field is predominantly in the $-\hat{\boldsymbol{r}}$ direction over the 1 ern polar cap, $\frac{d \Phi}{d t}<0$.

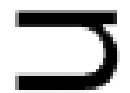

The largest contribution to the integral around the PCB is the dawn-dusk electric field $\mathbf{E}_{\mathrm{PC}}$ along distance $L_{\mathrm{PC}}$ such that,

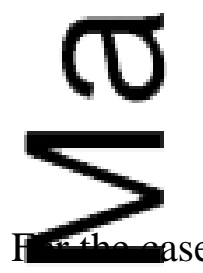

$$
\oint \boldsymbol{E} \cdot d \boldsymbol{l} \approx E_{P C} L_{P C}
$$

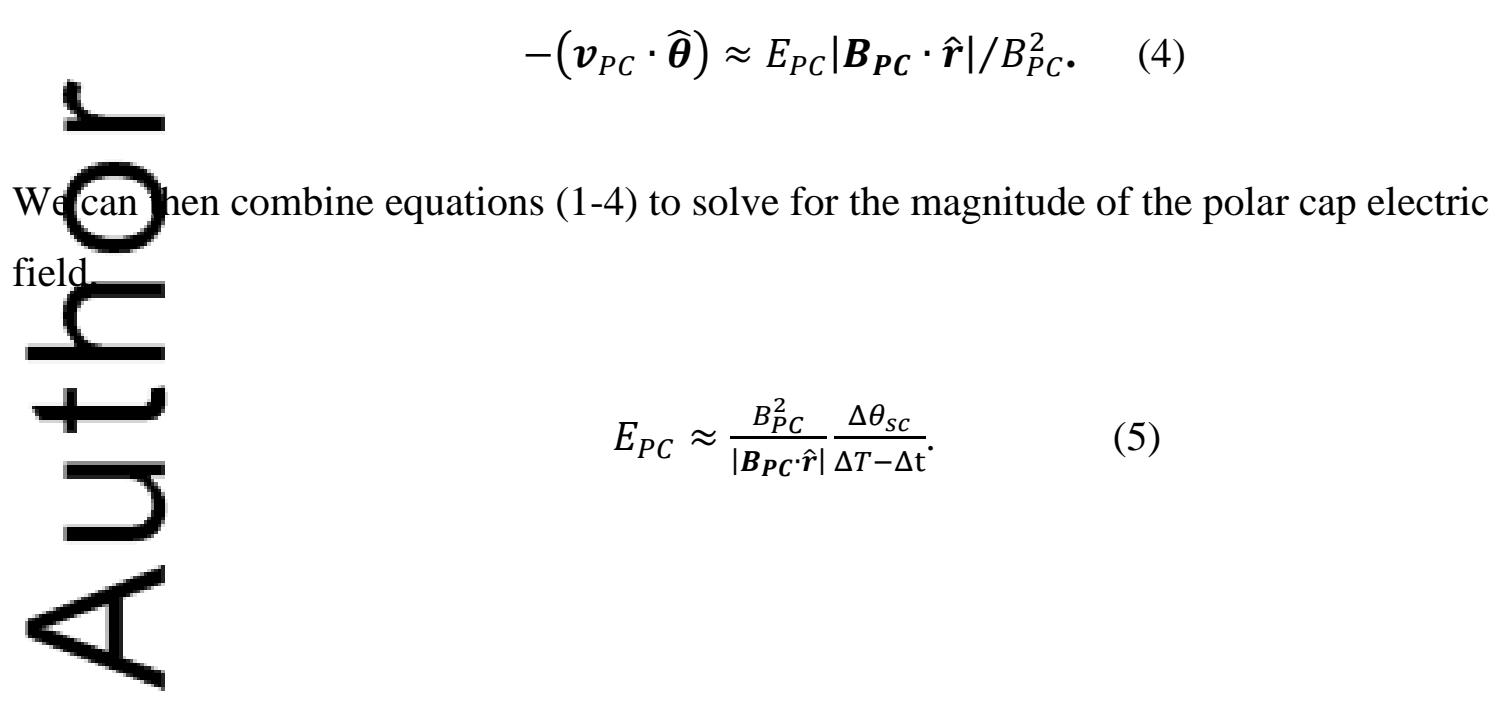


Finally, because the PCB electric field maps directly to magnetopause diffusion region as $E_{P C} L_{P C} \approx E_{R} L_{M P}$ (e.g., Toffoletto and Hill, [1989]), we can express the reconnection electric field as a function of measurements over the polar cap,

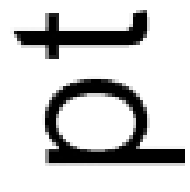

$$
E_{R} \approx \frac{B_{P C}^{2}}{\left|\boldsymbol{B}_{P C} \cdot \hat{\boldsymbol{r}}\right|} \frac{R_{M} \Delta \theta_{S C}}{\Delta T-\Delta \mathrm{t}}\left(\frac{L_{P C}}{L_{M P}}\right)
$$

For a PCB latitude of $\sim 60^{\circ}$ and a magnetopause standoff distance of $\sim 1.5 R_{\mathrm{M}}$, we use $L_{\mathrm{PC}} / L_{\mathrm{MP}} \sim 1 / 3$. This value assumes that the azimuthal extent of the dayside reconnection aloforesenting the magnetopause is approximately equal to the extent of the ${ }^{\mathrm{PQ}} \mathrm{S}$ The time history of relative energetic electron flux, $B_{\theta} / B$, and the reconnection electric ield calculated from equation (6) are shown in Figure 4 for two successive cro orrgs of Mercury's PCBL during the June 2011 SEP event. Changes in the magnetic fielar arrection $\left(B_{\theta} / B\right)$ are small $\left(<5^{\circ}\right)$ and are largely uncorrelated with the $\sim 5 \mathrm{~s}$ variation in ngtic electron fluxes. We conclude that the observed PCBL signatures are not the reanltafPB waves but rather correspond to time changes in the rate of magnetic reconnetion as illustrated in Figure 3. The mean and standard deviation of $\Delta t$ and $\Delta T$ calculated from the set of observed fluctuations are $6.7 \pm 2.7 \mathrm{~s}$ and $8.8 \pm 3.4 \mathrm{~s}$, respectively. These times result in derived reconnection electric fields that range from $E_{\mathrm{R}} \sim 0.3-3$ $\mathrm{mV} / \mathrm{m}$. solar wind speed of $\sim 300 \mathrm{~km} / \mathrm{s}$ was estimated from FIPS data using adjacent orb the plasma peak energy could be observed over the energetic particle fluxes [Gersnman et al., 2012]. This speed combined with an IMF strength of $\sim 50 \mathrm{nT}$ observed outside of the bow shock results in an estimated solar wind electric field $\left(E_{\mathrm{SW}}\right)$ of $\sim 15$ $\mathrm{mV} / \mathrm{m}$. The derived maximum instantaneous and average relative reconnection rates $\left(E_{\mathrm{R}} / E \rightarrow\right.$ are therefore $\sim 0.2$ and $\sim 0.02$, respectively. Because the spacecraft is a singleporm neasurement, the true reconnection rate for these intervals is likely between our 
reported average and instantaneous rates. Nonetheless, the apparent reconnection rate varies on the order of the ion gyroperiod at the magnetopause, i.e., a few seconds. Such modulations are consistent with particle-in-cell simulations of both symmetric and asymmetric magnetic reconnection, where small-scale structure and secondary islands cantemporarily disrupt or enhance the rate of reconnection [Daughton et al., 2006; Ka imabadi et al., 2007].

- Dayside FTEs have been observed at Mercury with similar time scales as these PCW flyctuations, and some flux-rope-like structures are observed near the ma hetopause for these orbits. We also observe a cusp filament in Figure 4a at 12:21:30 UT, a magnetic structure of diamagnetic origin, whose presence has been previously con ated with high FTE activity; it was proposed that these cusp filaments are the polar cap 1000 points of FTEs in the dayside magnetopause [Slavin et al., 2014; Poh et al., MESSENGER Observations of Cusp Plasma Filaments at Mercury, submitted to $J$. Ge Rhys. Res.: Space Phys.]. Our determination of the open-closed boundary is indenand of the magnetic field measurements such that we do not expect to find a oneto-Ond orrespondence of cusp filaments for each PCBL structure. Our observations sugsest that the contribution of bursty dayside reconnection at Mercury is likely derestimated for any given MESSENGER polar cap transit when using magnetic field signatures alone. Based on the duration of observed events and their $\mathbf{E x B}$ drift velocities, we stimate a latitudinal spatial extent ranging from $25 \mathrm{~km}$ to $100 \mathrm{~km}$. These spatial sca esmapped to the MP correspond to ion inertial scale lengths in Mercury's magnetic diffuston region.

Circular magnetic footpoints for these structures at the sampled altitudes would enoomnss magnetic fluxes of $\sim 10^{-3} \mathrm{MWb}$. This flux is an order of magnitude smaller than estimates of the flux carried by a single FTE [Imber et al., 2014]. Because the mreasured PCBL fluctuations provide constraints on latitudinal extent of these res, this discrepancy suggests that either the footpoint of an FTE in the polar cap is 
elongated in local time or a complex open-closed topological structure exists inside each flux rope such that multiple observed PCBL events map to a single FTE. In twodimensional reconnection, there is a clearly defined separatrix that prohibits the formation of such topology. However, fully three-dimensional simulations can produce cortplex topological domains at the smallest ion spatial scales [Dorelli et al., 2009]. So e vability in energetic particle fluxes inside the FTE shown in Figure 1 suggests that such fine-scale features could be present. Regardless of the PCBL structure being spalialor temporal in nature, we are probing the smallest ion scales relevant to rec nnegtion physics at Mercury.

\section{Doncluding Remarks}

Iercury's small magnetosphere is a sensitive magnetic reconnection laboratory. Energetic electrons observed by FIPS on MESSENGER during SEP events provide val able field-line tracers that map Mercury's magnetospheric topology at high cadence. Enhard SEP fluxes observed within an FTE provide confirmation that such structures are 1 hanism of open-flux transport at Mercury. In addition, quick changes in masetic topology were observed near the PCB for two successive orbits that correspond - kinetic scales and may provide a proxy for the time history of magnetic reconnection at the magnetopause. Such temporal variations are not easily measured at Ea h-measurements from spacecraft constellations (e.g., Cluster, THEMIS, MMS) can onl Tegnstruct the properties of the magnetic diffusion region in a single instant rather than provide time history of reconnection rate. Our analysis suggests that magnetic rec nnection at Mercury may be a fundamentally bursty process, where strong morlulations of the reconnection rate occur at ion gyroperiod scales.

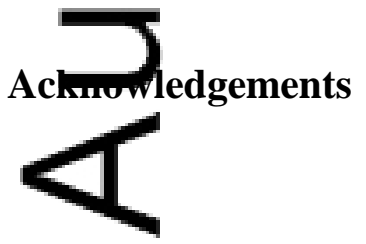


The authors wish to acknowledge the MESSENGER team, in particular the FIPS and MAG instrument teams for their efforts in producing fields and particle data at Mercury. MESSENGER data used in this study are available from the Planetary Data System (http://pds.nasa.gov). Uncorrected FIPS data (ESS-E/V/H/SW-EPPS-2-FIPSRALWDATA-V2.0) were used to estimate relative energetic particle fluxes. G.A.D is supported by a NASA Postdoctoral Program appointment at the NASA Goddard Space Flight_enter, administered by Oak Ridge Associated Universities through a contract withASA. This work was also supported by the NASA Discovery Data Analysis Program under grant NNX15AK88G.

(

References

Anderson, B. J., et al. (2007), The Magnetometer instrument on MESSENGER, Space ct. Kev., 131, 417-450.

An epson, B. J., C. L. Johnson, H. Korth, M. E. Purucker, R. M. Winslow, J. A. Slavin, Sn Solomon, R. L. McNutt Jr., J. M. Raines, and T. H. Zurbuchen (2011), The magnetic field of Mercury from MESSENGER orbital observations, Science, 35y $1859-1862$, doi:10.1126/science.1211001.

Anderson, B. J., C. L. Johnson, H. Korth, J. A. Slavin, R. M. Winslow, R. J. Phillips, R. L. McNutt Jr., and S. C. Solomon (2014), Steady-state field-aligned currents at Mercury, Geophys. Res. Lett., 41, 7444-7452, doi:10.1002/2014GL061677.

An rew, G. B., et al. (2007), The Energetic Particle and Plasma Spectrometer instrument Onime MESSENGER spacecraft, Space Sci. Rev., 131, 523-556.

C

Eaugnton, W., J. Scudder, and H. Karimabadi (2006), Fully kinetic simulations of

hdriven magnetic reconnection with open boundary conditions, Phys. Plasmas, $13,072101-1-072101-15$.

Dorelli, J. C., and A. Bhattacharjee (2009), On the generation and topology of flux transfer events, J. Geophys. Res., 114, A06213, doi:10.1029/2008JA013410. 
Dungey, J. W., Interplanetary magnetic fields and the auroral zone, Phys. Rev. Lett., 6, 47, 1961.

Eather, R. H., and S.-I. Akasofu (1969), Characteristics of polar cap auroras, J. Geophys. Res., 74, 4794-4798, doi:10.1029/JA074i019p04794.

Fetustern, Y. I., G. V. Starkov, Dynamics of auroral belt and polar geomagnetic Cisturbances, Planet. Space Sci., 15, 209, 1967.

Gershman, D. J., T. H. Zurbuchen, L. A. Fisk, J. A. Gilbert, J. M. Raines, B. J. Anderson, C. W. Smith, H. Korth, and S. C. Solomon (2012), Solar wind alpha particles and heavy ions in the inner heliosphere observed with MESSENGER, $J$. Ceophys. Res., 117, A00M02, doi:10.1029/2012JA017829.

Gerghnn, D. J., et al. (2015), MESSENGER observations of solar energetic electrons ithin Mercury's magnetosphere, J. Geophys. Res. Space Physics, 120, 85598571, doi:10.1002/2015JA021610.

Imber, S. M., J. A. Slavin, S. A. Boardsen, B. J. Anderson, H. Korth, R. L. McNutt Jr., and S. C. Solomon (2014), MESSENGER observations of large dayside flux wansfer events: Do they drive Mercury's substorm cycle?, J. Geophys. Res. Space Physics, 119, 5613-5623, doi:10.1002/2014JA019884.

Joh (n) C. L., et al. (2012), MESSENGER observations of Mercury's magnetic field structure, J. Geophys. Res., 117, E00L14, doi:10.1029/2012JE004217.

Karimavadi, H., W. Daughton, and J. Scudder (2007), Multi-scale structure of the Electron diffusion region, Geophys. Res. Lett., 34, L13104, doi:10.1029/2007GL030306.

Leppring, R. P., Jones, J. A., and L. F. Burlaga (1990), Magnetic field structure of Oterplanetary magnetic clouds at 1 AU, J. Geophys. Res., 95, 11,957-11,965.

Milan_S E. and J. A. Slavin (2011), An assessment of the length of variability of Mercury's magnetotail, Planet. Space Sci., 59, 2058-2065, doi:

Tـ1016/j.pss.2011.05.007.

Pand N. (1957), Sweet's mechanism for merging magnetic fields in conducting Guids, J. Geophys. Res., 62, 509-520, doi:10.1029/JZ062i004p00509. 
Russell, C. T., and R. C. Elphic (1979), ISEE observations of flux transfer events at the dayside magnetopause, Geophys. Res. Lett., 6, 33-36, doi:10.1029/GL006i001p00033.

Slavin, J. A., et al. (2009), MESSENGER observations of magnetic reconnection in Mercury's magnetosphere, Science, 324, 606-610, doi:10.1126/science.1172011.

Sravin, J. A., et al. (2010a), MESSENGER observations of large flux transfer events at Tergyry, Geophys. Res. Lett., 37, L02105, doi: 10.1029/2009GL041485.

Slavin _ A., et al. (2010b), MESSENGER observations of extreme loading and nloading of Mercury's magnetic tail, Science, 329, 665-668, dor.10.1126/science.1188067.

s. A. et al. (2012), MESSENGER observations of a flux-transfer-event shower at ()$^{n}$

Slavin_ A., et al. (2014), MESSENGER observations of Mercury's dayside magn tosphere under extreme solar wind conditions, J. Geophys. Res. Space Physics, П9, 8087-8116, doi:10.1002/2014JA020319.

Sis oe, G. L., and T. S. Huang (1985), Polar cap inflation and deflation, J. Geophys. Res., 90, 543-547, doi:10.1029/JA090iA01p00543.

son

xplorer 12 observations, J. Geophys. Res., 72, 171-183, Soi:10.1029/JZ072i001p00171.

Sweet, P. A., The neutral point theory of solar flares, Electromagnetic Phenomena in Cosmical PhysicsB. Lehnert, 123-134, Cambridge University Press, London, 1958.

To foleto, F. R., and T. W. Hill (1989), Mapping of the solar wind electric field to the Earth's polar caps, J. Geophys. Res., 94, 329-347,

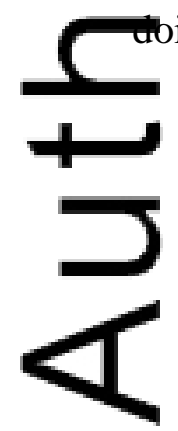


Troshichev, O. A., E. M. Shishkina, C.-I. Meng, and P. T. Newell (1996), Identification of the poleward boundary of the auroral oval using characteristics of ion precipitation, J. Geophys. Res., 101, 5035-5046, doi:10.1029/95JA03634.

Winningham, J. D., and W. J. Heikkila (1974), Polar cap auroral electron fluxes observed with Isis 1, J. Geophys. Res., 79, 949-957, doi:10.1029/JA079i007p00949

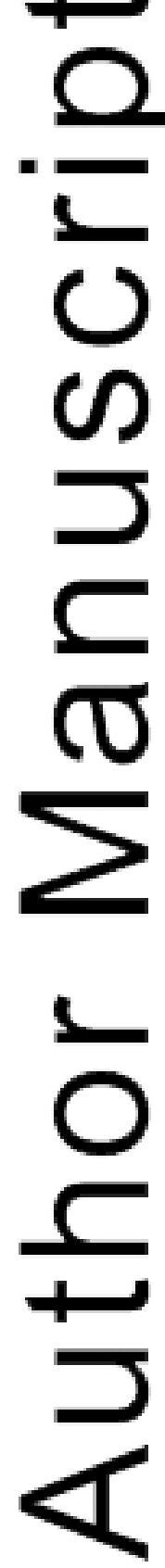

This article is protected by copyright. All rights reserved. 


\section{Figures and Captions}

Figure 1. (a) Sketch of MESSENGER trajectory through an FTE observed on the dayside magnetosphere (LT $1500 \mathrm{hr}$, Alt $1660 \mathrm{~km}$ ) on 21 September 2012. A helical flux rope is embedded in Mercury's magnetospheric field. (b) Relative energetic particle flux as inferred by FIPS and magnetic field vectors from MAG in minimum variance coordinates W1tr unit vectors in the (c) minimum variance direction: [-0.05, 0.20, 0.98], (d) inte ime late variance direction: $[-0.96,-0.29,0.01]$, and (e) maximum variance direction: [0.10_02, 0.20] in MSM coordinates with eigenvalues 1.8,56.7, and 154.5, respectively. The FIPS measured energetic particle rates are normalized by the average value in the dayside magnetosheath. Increased fluxes are observed inside the core of the FTE (snrded region) indicate that it transports open magnetic field. Gaps in the reported SE flun indicate time periods between FIPS energy scans in which the sensor was not accurating data.

Figu. (a) Illustration of MESSENGER descending trajectory over Mercury's polar cap for a June 2011 SEP event. Open and closed magnetic field regions are indicated with red and lue lines, respectively. (b) Relative energetic particle flux as inferred by FIPS and(c) magnetic field vectors from MAG during 8 June 2011. The FIPS measured ene getre particle rates are normalized by the average value in the dayside madnesheath. In the dayside closed-field region, fluxes drop significantly from their magnetosheath and polar cap levels. Fluctuations in SEP flux appear at 00:26 UT with no 0 re ponding large-amplitude fluctuations in the magnetic field data are labeled as the PCBL.

Figurs. Top-view illustration of MESSENGER trajectory over Mercury's polar cap. (a) er the open-field region, FIPS measures high fluxes of energetic particles. (b) Fluxes drop sharply when the spacecraft enters the closed-field region. (c) If magnetic recgnnection occurs at the magnetopause over length scale $L_{\mathrm{MP}}$, the PCB expands by azimutral length $L_{\mathrm{PC}}$ and polar length $D_{\mathrm{PC}}$. If sufficient flux has reconnected to overtake the paecraft, high SEP fluxes return until (d) the spacecraft once again exits the openfiel reo on. Times between adjacent rises and falls of measured SEP flux provide information on the duration and strength of dayside magnetic reconnection.

Figure 4. PCBL transits of MESSENGER for two successive orbits on (a) 7 June 2011 aros June 2011. The top panels show high cadence $(\sim 150 \mathrm{~ms})$ energetic particle fluxacom FIPS normalized to their MSH values. Using the timing of the observed bursts in SEP flux, the reconnection electric field at the magnetopause is estimated in the bottom panels. Bursts of $\sim 0.3-3 \mathrm{mV} / \mathrm{m}$ fields correspond to peak and average ection rates of $\sim 0.2$ and 0.02 , respectively. 


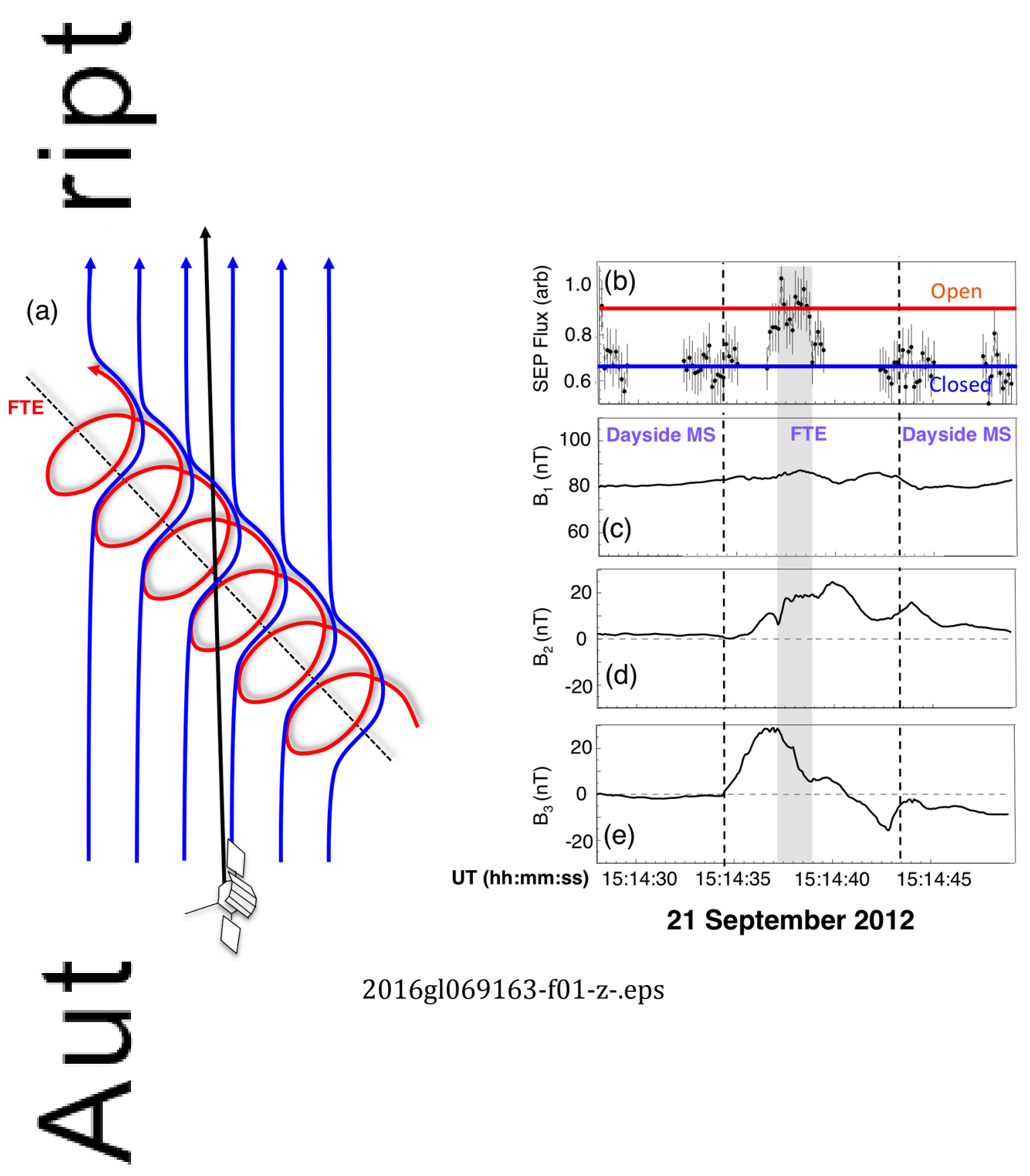

This article is protected by copyright. All rights reserved. 

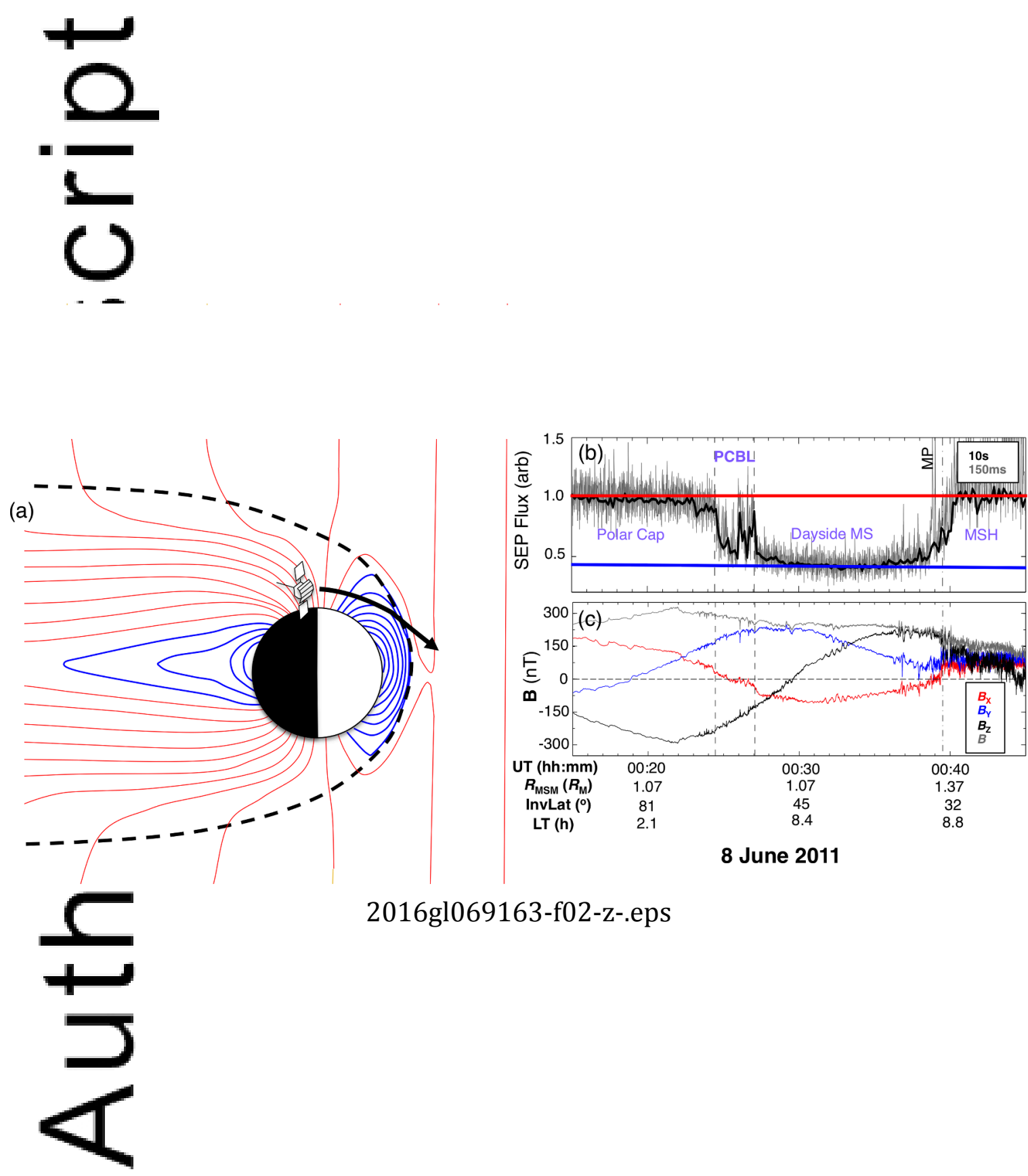

This article is protected by copyright. All rights reserved. 


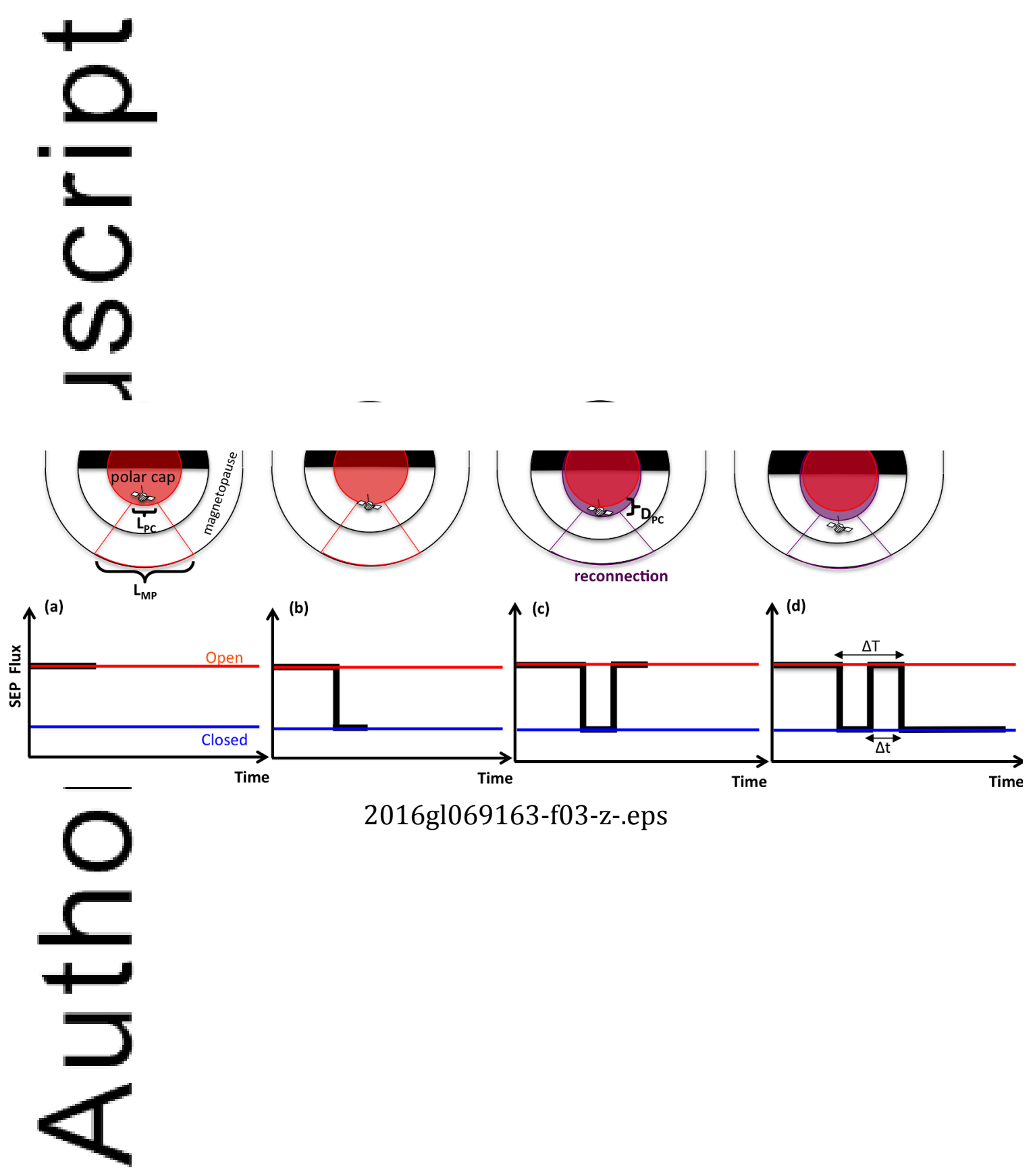

This article is protected by copyright. All rights reserved. 


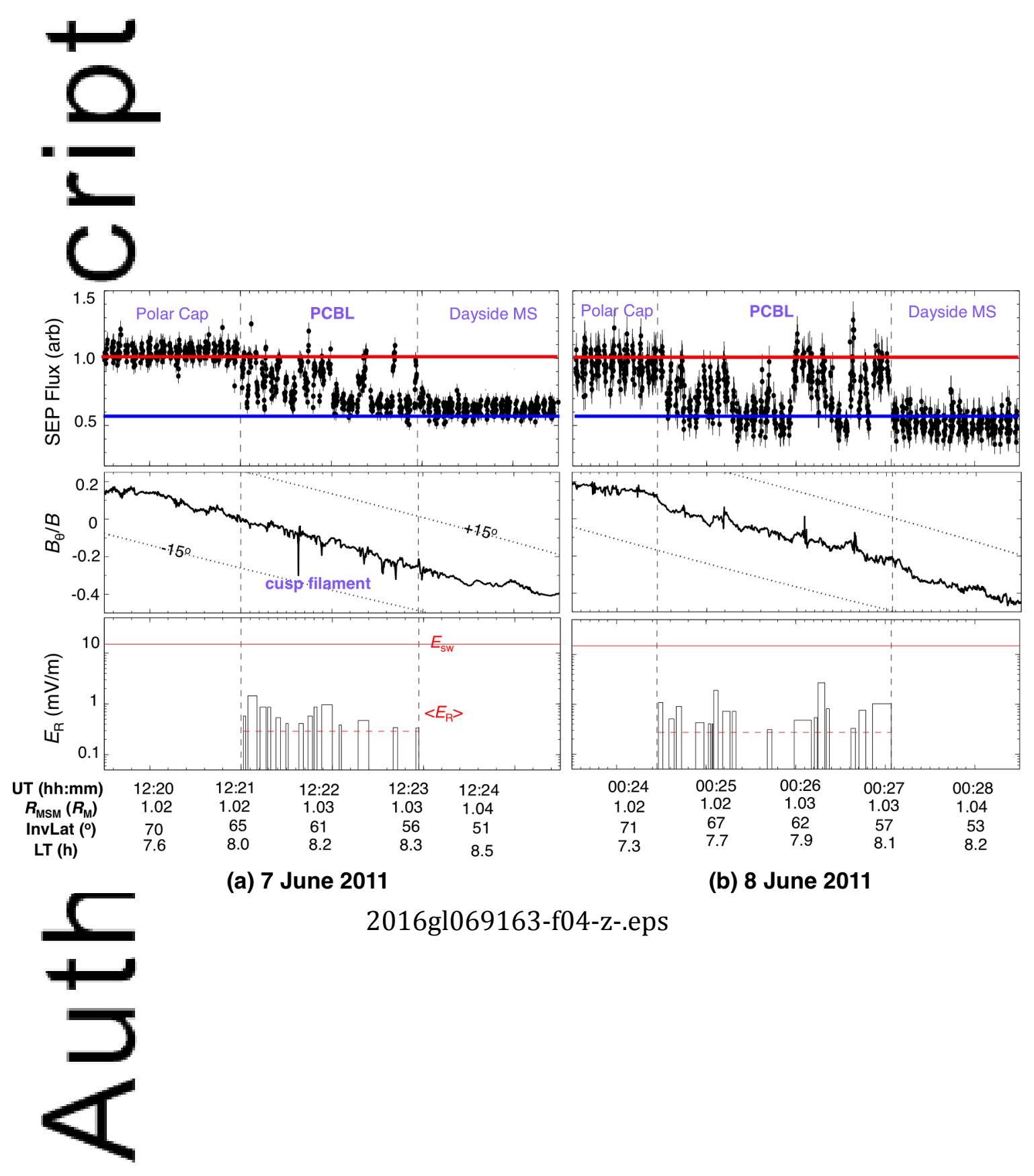

This article is protected by copyright. All rights reserved. 